Brit. J. vener. Dis. (1959), 35, 20.

\title{
POSSIBLE SIGNIFICANCE OF PPLO IN HUMAN GENITAL INFECTION*
}

\author{
BY \\ E. KLIENEBERGER-NOBEL \\ The Lister Institute of Preventive Medicine, London
}

The organism causing pleuropneumonia in cattle, still a devastating disease in South Africa and parts of Australia, was discovered in 1898 by a group of French workers. In 1923 a very similar organism was found to be the cause of another animal disease, agalactia of sheep and goats. Since then a considerable number of organisms of the same group, some pathogenic, others saprophytic, have been discovered by various workers. It has thus emerged that a whole family of microbes exists, differing from both bacteria and viruses. Some of these play an important part in the aetiology of disease in animals and probably also in humans.

The organisms possess very small filterable elements, not larger than the elementary bodies of the pox viruses. In addition, they possess larger forms of varying sizes and shapes, which have no rigid cell walls. Unlike the viruses they can be grown on artificial media of special constitution. On solid media their colonies are very small (10 to $500 \mu$ in diameter) and have a characteristic appearance with a dense centre, embedded in the medium, and a lighter, superficially spreading, peripheral zone.

PPLO also have a peculiar mode of producing infection, which follows a similar pattern in the various diseases caused by organisms of the group. A few instances may suffice to demonstrate this. Agalactia is a seasonal disease which affects the ewes only after lambing. Lactation stops and the animals produce a purulent secretion instead of milk. They probably become infected while grazing in fields contaminated by other herds, but symptoms only develop at lambing time.

Bronchopneumonia occurs widely in laboratory rats as well as in wild rats. It can be acute, but very often takes a slow and chronic course. Very old rats invariably show severe lung lesions which are usually

* Paper read to the Medical Society for the Study of Venereal Diseases at Cambridge, July 4, 1958. restricted to one or two lobes. Klieneberger and Steabben $(1937,1940)$ examined a large number of stock rats at the Lister Institute during the years 1935 to 1937 . They found that very young rats were free from lesions and PPLO were only rarely isolated from their lungs. In older animals both PPLO and lung lesions were found in increasing numbers. In young rats, initially without lung lesions, the disease developed rapidly following ligation of a bronchus (Klieneberger-Nobel and Cheng, 1955); 2 days after the operation PPLO were already found in the lungs and their number increased with the progress of the lesions. Since the disease becomes manifest in every animal after operation, the organisms must have been latently present at the time the ligature was performed. It appears that the operation was necessary to produce conditions suitable for the organisms to gain a foothold in the lungs, where they multiplied thereafter and caused severe disease. Though certain conditions are apparently essential for the outbreak of the disease the significance of the organisms in establishing it cannot be doubted. Even in a disease so fulminating as pleuropneumonia of cattle we do not know the accessory factors necessary, besides the organisms, to start the disease. This is borne out by the fact that attempts to produce the disease artificially, for example with a heavy spray of viable, pathogenic organisms, have very often been unsuccessful.

It is well known that PPLO are frequently found in humans. Organisms different from the human genital type occur ubiquitously in the mouth and seem to be harmless saprophytes. In contrast to this, it has often been suggested that PPLO isolated from human genitals are pathogenic. The first report of such an isolation was by Dienes and Edsall (1937). Since then the frequent presence of PPLO in the discharge from non-gonococcal urethritis has caused particular interest. 
Stokes (1955) isolated PPLO from the blood of a woman with puerperal fever. She also found the organisms in the wound and pleural fluid of a patient suffering from post-operative empyema. She further isolated PPLO from high vaginal swabs in a second case of puerperal fever and in a case of hydrosalpinx with pyrexia. Stokes reported: "In three of the patients specific antibody developed during the infection, reached a maximal titre at about 3 weeks during convalescence, and fell thereafter." The author considered the presence of the organisms to be significant since they occurred in pure culture and a rising titre of specific antibody was demonstrated.

The cultural and serological work reported here (Klieneberger-Nobel, 1959; Card, 1959) was undertaken with a view to collecting further evidence on the significance of PPLO in pathological genital conditions and particularly in non-gonococcal urethritis (N.G.U.) in men. It should be pointed out that N.G.U. is not precisely defined. The term is applied to all cases of urethritis in which gonococci are absent, and it is well known that it can have various causes among which PPLO infection could be only one. If therefore PPLO could be demonstrated in a substantially higher proportion of N.G.U. cases than in healthy males their significance would be strongly suspected.

The methods for the cultivation of PPLO from human genitals have been described elsewhere (Klieneberger-Nobel, 1959). Male patients suffering from N.G.U. and females with various diseases of the genital organs from the V.D. clinics of St. Mary's Hospital and the London Hospital were examined. A group of pregnant women and another of gynaecological patients were also investigated. Specimens from the anterior urethra in males and from the vagina, cervix, and urethra in females were collected with a platinum loop and spread on special media without delay. In cases of Reiter's disease, synovial fluids were also examined, but PPLO were never isolated from them. In healthy males, urine was used as a specimen, since scraping the urethra does not provide a satisfactory specimen when no discharge is present. In a preliminary investigation it was first established that human genital PPLO remained viable in urine for at least 6 hours (Blyth) and secondly, that PPLO were isolated with the same frequency from urine as from discharge.

\section{Cultural Tests}

In the course of the investigation 98 strains of PPLO were isolated from human genitals. Their growth characteristics showed great similarity. A large proportion of these strains was studied serologically by means of a complement-fixation reaction (Card, 1959). It was thus shown that the examined strains belonged to a single serological group.

Men.-It was necessary to find a group of persons free from genital infection as controls. We assumed that boys under the age of puberty and men of a high hygienic standard and free from symptoms would represent such controls. Of 47 specimens of urine (see Table I) from boys under the age of 13 none was positive. From 100 specimens collected from members of the staff of the hospitals three yielded PPLO. One of the men was shown to have N.G.U., another had no symptoms but reported previous attacks of prostatitis, and no inquiry was made in the third case. We conclude that PPLO are rare in the urethra of healthy men and that boys under the age of puberty are free from them.

Table I shows that very different results were obtained in our study of male patients from the two venereal disease clinics. PPLO were isolated from

TABLE I

INCIDENCE OF PPLO IN MALES

\begin{tabular}{|c|c|c|c|c|c|c|c|c|}
\hline \multirow{2}{*}{\multicolumn{5}{|c|}{ Groups of Subjects }} & \multirow{3}{*}{$\begin{array}{c}\text { Number of Cases } \\
200\end{array}$} & \multicolumn{2}{|c|}{ PPLO Positive } & \multirow{3}{*}{ Specimens Examined } \\
\hline & & & & & & \multirow{2}{*}{$\frac{\text { No. }}{57}$} & \multirow{2}{*}{$\frac{\text { Per cent. }}{25 \cdot 5}$} & \\
\hline Total & $\cdots$ & $\cdots$ & $\cdots$ & $\cdots$ & & & & \\
\hline Acute N.G.U. & $\cdots$ & $\cdots$ & $\cdots$ & $\cdots$ & 65 & 31 & 48 & \multirow{6}{*}{ Discharge and Scrapings } \\
\hline Untreated Gonc & orrhoea & $\cdots$ & $\cdots$ & $\cdots$ & 49 & 15 & 30 & \\
\hline \multicolumn{3}{|c|}{ Miscellaneous Conditions* } & $\cdots$ & $\cdots$ & 43 & 4 & $9 \cdot 3$ & \\
\hline Reiter's Disease & $\quad \cdots$ & $\cdots$ & $\cdots$ & $\cdots$ & 20 & 2 & 10 & \\
\hline Chronic N.G.U. & $\cdots$ & $\cdots$ & $\cdots$ & $\cdots$ & 17 & $\mathbf{0}$ & 0 & \\
\hline Cured N.G.U. & $\cdots$ & . & .. & $\cdots$ & 7 & 0 & 0 & \\
\hline \multirow{2}{*}{ Controls } & Health & Ad & $\cdots$ & $\cdots$ & 100 & 3 & 3 & \multirow{2}{*}{ Urine } \\
\hline & \multicolumn{4}{|c|}{ Boys under 13 years of age } & 47 & $\mathbf{0}$ & $\mathbf{0}$ & \\
\hline
\end{tabular}

* Epididymitis, chronic and low grade prostatitis, penile sore, warts, and nine symptomless cases. 
57 ( $25 \cdot 5$ per cent.) of a total of two hundred patients. When these patients were grouped on clinical grounds it was found that 31 (48 per cent.) out of 65 patients with acute N.G.U. harboured PPLO. Of 49 patients with acute, untreated gonorrhoea fifteen ( 30 per cent.) harboured PPLO. Among the other patients suffering from Reiter's disease or miscellaneous conditions of the genitals, 10 per cent. yielded PPLO. The highest proportion of PPLO isolations was therefore found in the acute N.G.U. group.

Women.-As reported by various authors, PPLO occur more frequently among female V.D. clinic patients than among males. Cultural examination for PPLO was positive in 33 ( 56 per cent.) of a total of 59 women. In untreated and treated gonorrhoea and in cervicitis, vaginitis, or urethritis, the percentages of PPLO isolations were very high (between 80 and 90 per cent.). PPLO also occurred together with Trichomonas vaginalis (two out of six cases positive). However, in fourteen sypmtomless women and in three who were suffering only from warts or genital ulcers, PPLO were not found. Approximately 20 per cent. of 51 obstetric and 58 gynaecological patients attending the London Hospital carried PPLO, but several of them had trichomonal vaginitis or thrush.

Consorts.-In four pairs of consorts examined, both partners carried PPLO; in three pairs the organisms were not found in either partner.

The reported data show that, in men, PPLO are associated with acute N.G.U., and in women with cervicitis, vaginitis, and urethritis. They are also frequently associated with gonococcal infection and trichomonal infestation. They are extremely rare in healthy men and women and in boys under the age of puberty. Their transfer by sexual intercourse is likely.

\section{Serological Tests}

It has been shown in rats that the presence of a pathogenic PPLO produces a rising antibody titre as measured by complement fixation tests (Card, 1959). This titre remains between $1: 2000$ and $1: 5000$ for some time even after the symptoms have subsided. As mentioned before, antibodies against the human genital type of PPLO have been demonstrated in infected humans. If the presence of PPLO in the human urogenital tract leads to antibody production, then the incidence of antibodies in any group of people should be related to the probability of infection. The groups of subjects expected to have various incidences of venereal disease are listed in Table II. As controls free from venereal disease, blood donors and children were examined. A high proportion of subjects with present or past venereal infections could be expected among V.D. clinic patients. As intermediate groups, medical outpatients as well as people attending ante-natal and gynaecological departments were examined. The methods used for the complement-fixation test and for the preparation of antigens have been described elsewhere (Card, 1959). The results of the tests are summarized in Table II. A total of 700 patients from the two V.D. clinics was examined, and 34 per cent. of these had PPLO antibodies in their serum. Women gave approximately twice as many positive results as men. The very high percentage of positive sera in the V.D. clinic patients corresponds closely to the positive cultural tests. The serological data further tally with our cultural findings as regards the frequency of PPLO infection in men and women. In contrast to these results, it was found that sera containing PPLO antibodies were very rarely found among the two control groups of children and blood donors. The percentage of subjects with positive titres was also low among medical out-patients and women attending an ante-natal clinic in a country district where the incidence of V.D. is expected to be

TABLE II

DISTRIBUTION OF PPLO ANTIBODIES IN HUMAN SERA

\begin{tabular}{|c|c|c|c|c|c|c|c|c|c|c|c|c|}
\hline \multirow{2}{*}{\multicolumn{7}{|c|}{ Groups of Subjects }} & \multicolumn{2}{|c|}{ Total } & \multicolumn{2}{|c|}{ Males } & \multicolumn{2}{|c|}{ Females } \\
\hline & & & & & & & \multirow{2}{*}{$\begin{array}{c}\text { No. } \\
\text { Tested }\end{array}$} & \multirow{2}{*}{$\begin{array}{c}\begin{array}{c}\text { Positive } \\
\text { (percentage) }\end{array} \\
34\end{array}$} & \multirow{2}{*}{$\begin{array}{c}\begin{array}{c}\text { No. } \\
\text { Tested }\end{array} \\
284\end{array}$} & \multirow{2}{*}{$\begin{array}{c}\begin{array}{c}\text { Positive } \\
\text { (percentage) }\end{array} \\
18 \cdot 7\end{array}$} & \multirow{2}{*}{$\begin{array}{c}\begin{array}{c}\text { No. } \\
\text { Tested }\end{array} \\
416\end{array}$} & \multirow{2}{*}{$\begin{array}{c}\begin{array}{c}\text { Positive } \\
\text { (percentage) }\end{array} \\
44.5\end{array}$} \\
\hline V.D. Patients & $\ldots$ & $\cdots$ & $\ldots$ & . & . & . & & & & & & \\
\hline Medical Out-pa & tients & $\ldots$ & $\ldots$ & $\cdots$ & . & . & 198 & $6 \cdot 1$ & 111 & $4 \cdot 5$ & 87 & $8 \cdot 1$ \\
\hline \multicolumn{5}{|c|}{ Antenatal Patients (Country District) .. } & . & . & - & - & - & - & 96 & $6 \cdot 3$ \\
\hline \multicolumn{5}{|c|}{ Gynaecological Patients (London Hospital) } & . & . & - & - & - & - & 44 & $20 \cdot 4$ \\
\hline Blood Donors & $\cdots$ & . & . & $\cdots$ & . & . & 297 & $2 \cdot 1$ & 188 & $1 \cdot 1$ & 109 & $3 \cdot 7$ \\
\hline Children & . & $\ldots$ & $\ldots$ & $\cdots$ & $\ldots$ & $\cdots$ & 104 & 2 & 72 & $2 \cdot 8$ & 32 & - \\
\hline
\end{tabular}


low. However, it was slightly higher than that of the blood donors and children. The gynaecological patients (London Hospital) occupy an intermediate position.

The serological findings show that positive titres are frequent in those groups of subjects in which venereal infections are rife, and in which PPLO have also been cultured most commonly. In rats antibody titres occur only when the animals are infected with pathogenic organisms. It is therefore assumed that specific antibody against PPLO in humans also reveals present or past PPLO infection.

\section{REFERENCES}

Blyth, W. (unpublished data).

Card, D. H. (1959). Brit. J. vener. Dis., 35, 27.

Dienes, L., and Edsall, G. (1937). Proc. Soc. exp. Biol. (N.Y.), 36, 740. Klieneberger, E., and Steabben, D. B., (1937). J. Hyg. (Camb.), 37, 143. KI (1940). Ibid., 40, 223.

Klieneberger-Nobel, E. (1959). Brit. med. J., 1, 19.

and Cheng, K. K. (1955). J. Path. Bact., 70, 245.

Stokes, E. J. (1955). Lancet, i, 276. 